\title{
Labyrinthe
}

22 | 2005 (3)

La Biopolitique (d')après Michel Foucault

\section{De la vie dans la vie : sur une étrange opposition entre zôê et bios}

\section{Laurent Dubreuil}

\section{(2) OpenEdition \\ 1 Journals}

\section{Édition électronique}

URL : http://journals.openedition.org/labyrinthe/1033

DOI : $10.4000 /$ labyrinthe.1033

ISSN : 1950-6031

Éditeur

Hermann

\section{Édition imprimée}

Date de publication : 1 novembre 2005

Pagination : 47-52

\section{Référence électronique}

Laurent Dubreuil, «De la vie dans la vie : sur une étrange opposition entre zôe et bios », Labyrinthe [En ligne], 22 | 2005 (3), mis en ligne le 22 juillet 2008, consulté le 19 avril 2019. URL : http://

journals.openedition.org/labyrinthe/1033; DOI : 10.4000/labyrinthe.1033 


\section{DE LA VIE DANS LA VIE: sur une étrange opposition entre $z \hat{o} \hat{e}$ et bios}

Laurent DuBREUIL 1d79@cornell.edu

«Les Grecs ne disposaient pas d'un terme unique pour exprimer ce que nous entendons par le mot vie. Ils se servaient de deux mots [...]: $z \hat{o} \hat{e}$, qui exprimait le simple fait de vivre, commun à tous les êtres vivants (animaux, hommes ou dieux), et bios, qui indiquait la forme ou la façon de vivre propre à un individu ou à un groupe ${ }^{1} \gg$ Ces phrases de Giorgio Agamben ouvrent Le Pouvoir souverain et la vie nue. Elles ont aujourd'hui popularisé une distinction «grecque » au cœur même de la vie, que nous aurions perdue. Selon Agamben, l'opposition se marquait, dans la langue grecque, entre la « simple vie naturelle » et le «mode de vie particulier» (p. 9), « la vie en général et le mode de vie qualifié qui est propre aux hommes »(p.76), «vie privée et existence politique» (p. 201). Zôê, le premier terme, renverrait à une vie en partage avec l'ensemble du vivant, et que le devenir humain aurait à convertir en vie qualifiée, en eu zèn (bonne vie, voir p. 10 sqq.), en suzèn (vivre avec), bref en bios politikos. Au mieux, la zôê « a pour lieu propre la domus » (p. 201), espace circonscrit à quoi ne se résume pas la cité.

Cette structure conceptuelle irait de pair avec le fonctionnement du sémantisme en grec, la démocratie athénienne et la réflexion politique classique (surtout Platon et Aristote). La modernité, elle, aurait remis en cause la différence, l'écart entre zôê et bios. Historiquement parlant, «le fait décisif» tient en ce que «bios et zôê, droit et fait, entrent dans une zone d'indifférenciation irréductible » (p. 17), à l'origine de la biopolitique totalitaire de l'Occident.

L'analyse d'Agamben est notoire. Je la rappelle afin de souligner combien l'hypothèse linguistique est solidaire de tout le mouvement de démonstration de la trilogie Homo sacer. La séparation effective entre

1. Giorgio Agamben, Homo sacer, vol. I, Le Pouvoir souverain et la vie nue, Paris, Seuil, 1997, p. 9. 
vie nue et existence politique nous est confiée par les mots des Grecs. Ce point est désormais admis largement. Sur les sites web, l'association de zôê et bios donne un grand nombre de réponses. Dans un livre aussi sérieusement documenté que Bios, de Roberto Esposito², le couple bipolaire n'est pas mis en doute une seconde. Je disais qu'Agamben popularisait, car il renvoie à deux auteurs pour le début de son étude, Hannah Arendt et Foucault. Ce dernier est invoqué pour la conclusion de $L a$ volonté de savoir et les cours du Collège de France autour de la biopolitique. Arendt apparaît alors comme celle qui restaure la différence effacée. On trouve en effet dans The Human Condition que bios se situe " par opposition à la simple zôe », et qu'elle se révèle ainsi "vie spécifiquement humaine ${ }^{3} »$. Le jeu entre zôê et bios fut remarqué bien avant, par des locuteurs grecs. Les grammairiens ont proposé deux grandes conjectures pour expliquer la concurrence des vocables. Pour les uns, le distinguo tiendrait à une représentation de la durée et de la continuité. Bios serait «le temps de la zôe $\hat{e}^{4}$ », une portion particulière; à moins que ce ne fût l'inverse. La seconde explication est à la source des propos d'Arendt, d'Agamben, etc. Ammonius, un Alexandrin, estimait que «bios convient aux animaux à raison [logikôn zôôn], c'est-à-dire aux seuls êtres humains, zôê aux êtres humains et aux animaux sans raison ${ }^{5}$ ». Il faut aussitôt noter que le philologue utilise Aristote, sans se demander si l'usage rationnel que semble préférer le Stagirite a une efficace pour toute la langue grecque. Ajoutons qu' on a pu relever a contrario que bios était un terme revenant aux animaux non rationnels ${ }^{6}$. Chacune des deux constructions de répartition s'appuie évidemment sur des exemples, plus ou moins probants.

Lorsqu'elle opte pour une justification plutôt qu'une autre, Arendt demeure évasive. Il n'est pas certain qu'elle veuille se référer à un usage un du grec; elle cite d'ailleurs Aristote à l'appui. Sans doute le Philosophe est-il l'auteur où la distribution ne tient pas trop mal. Mais il serait bien rapide de croire que, même chez lui, bios soit « spécifique-

2. Bios. Biopolitica e filosofia, Einaudi, Turin, 2004.

3. Hannah Arendt, La Condition de l' homme moderne, Paris, Calmann-Lévy, 1961, p. 110.

4. J'utilise la mise au point du Thesaurus Grace Linguae, fin de l'article Bios. (Je traduis les textes grecs, ici et plus loin.).

5. Ammonius, De Adfinium vocabulorum differentia, Leipzig, Teubner, 1966, édition du texte grec Klaus Nickau, $\S 100$.

6. Rappelé par le Thesaurus..., loc. cit. 
ment humain » ou « politique ». Il est par exemple question de « vivre [zèn] la vie [bion] d'une plante ${ }^{7} » ;$ on lit aussi que « les différences entre animaux [zôôn] sont relatives à leurs vies [bious], leurs caractères et leurs organes $^{8} »$. Certes, dans les deux occurrences, on objectera qu'Aristote n'a pas voulu répéter un mot de la famille zôe ; mais cette maladresse conceptuelle due au seul souci d'un beau style ne convainc guère. Il se pourrait que le recours à bios (pour une plante) soit dû aux limitations internes du grec, où le verbe bioô est rare « en prose attique » (p. 10). Cela empêcherait le syntagme "vivre [biônai] la vie [zoên] d'une plante ». Je me contente de transposer ici la solution à un problème analogue que donne Agamben (l'expression théoriquement dérangeante zôon politikon). La rareté de bioô reste à prouver; et il existe de toute façon un autre verbe courant de même racine, bioteu $\hat{o}^{9}$. On le trouve dans Aristote décrivant des oiseaux qui ne volent pas, mais qui « vivent [bioteuousin] près de l'eau »: bios et non pas zôô... En fait, on peut suivre Agamben (plutôt qu'Arendt) sur le degré de qualification. Aristote aurait tendance à mettre bios davantage avec la vie particulière (existence dans la Cité, végétale, aquatique) et zôê dans le général. On aurait le jeu entre le substantif « le mode de vie qualifié » et un autre, plus large. Mais en aucun cas, bios n'est réservé aux humains, ni à l'exercice politique. Enfin, Aristote n'est pas le corpus grec. Truisme utile à redire. Quand il fait contraster bios et zôê, Aristote s'inscrit dans une tradition discursive. Nous ne prélèverons qu'un exemple diplopique. Dans le premier chœur d'Héraklès, Euripide invente une morale post mortem, où les bons, arrivés au terme de leurs ans, se verraient offrir une nouvelle chance, tandis que les mauvais seraient punis en mourant sans retour. Deux vers énoncent le dogme improbable: «La vilenie aurait une seule vie [biotan] en vie $[z \hat{a} a s]^{10} »$. Zôas est un génitif délicat à expliquer; il dit la vie de la vie. Les traducteurs décident souvent d'y voir un simple intensif, et laissent de côté le redoublement. Je choisis l'ambiguïté, de dire qu'une vie serait de la vie parce que dans la vie. Bios est portion définie, ou souscas, de zôê, un peu comme avec Aristote. Empressons-nous de citer une

\footnotetext{
7. Aristote, De Generatione animalium, II, 3, 736b.

8. Aristote, Historia animalium, I, 1, 487a.

9. Ammonius donne un nouveau distinguo entre bioô et bioteuô, qui ne tient pas davantage que le précédent (De Adfinium vocabulorum differentia, § 102).

10. Euripide, Héraklès, v. 663-664.
} 
autre phrase, qui désigne exactement la dynamique inverse. Voici un coup de Platon, qui, dans le Timée, imagine une migration psychique proche du chœur d'Euripide. Il fait mention d'une âme « qui a traversé une vie [zồn $]$ infirme en la vie [tou biou $]^{11} »$. Dernier échange de termes, où bios vaut cette fois pour la vie en général, et zôê pour le parcours singulier.

Si l'on entend rendre cohérents ces éléments disparates (et le fautil?), on ne saurait guère aller au-delà du repérage de tendances entre le plus général et le plus particulier; tout en gardant à l'esprit que les rôles ne sont pas répartis par avance. Car la langue, pour avoir ses structures, ses lois, n'est pas une donnée absolue et externe; le discours a encore à la réinventer singulièrement; ce que firent Euripide, Platon ou Aristote. Agamben, qui solidifie une conjecture alexandrine reprise par Arendt, a bien le droit de répartir un contenu conceptuel dans zôê à l'inverse de bios. Il participe en cela du moment socratique, quand le philosophe allait voir les Athéniens de la rue pour redéfinir avec eux le langage qu'ils parlaient. La difficulté ne vient donc pas de l'opération philosophique mais de sa justification historico-philologique. Agamben épure Aristote et il l'extrapole à l'entité merveilleuse « les Grecs ». À l'argument d'autorité, infondé comme on l'a vu, se combine un réseau de motifs tacites, qu'il convient d'exhiber, voire de contester. Alors même qu'il ancre toute « l'existence politique » dans le langage (logos, voir p. 16), Agamben reste sourd au phénomène sémantique dans la langue. La signification peut advenir dans un discours, où bios et zôê prennent des valeurs qui se commentent, se complètent ou se critiquent - loin de l'indexation des sens clos et rationalisés. La confusion entre la clarification conceptuelle de la définition et les usages des langues ou des discours mène à l'étai lexical de l'analyse politique. La fin du Pouvoir souverain... nous rappelle un personnage agissant depuis la coulisse: « Le bios gît aujourd'hui dans la zôê exactement comme, dans la définition heideggerienne du Dasein, l'essence gît (liegt) dans l'existence. » (p. 202). Au-delà de ce motif, Agamben rejoint Heidegger qui affirmait: «La langue grecque, et elle seule, est $\log o s^{12}$.» Comprendre le logos politique nécessite d'aller vers l'Ursprache philosophique que serait le grec. Cet idiome est à l'unisson du réel: deux mots désignent deux choses différentes (la vie

11. Platon, Timée, 44c.

12. Martin Heidegger, Was ist das - die Philosophie?, Pfullingen, Neske, 1956, p. 20; je traduis. 
nue, la vie politique). Agamben place apparemment la dé-chéance à la fin de l'Antiquité, quand pour Heidegger les tracas arrivent dès Socrate. Autre modification: à Fribourg, la célébration hellénique inclut la louange d'une langue à l'unisson de l'être, tandis qu'en Vénétie l'on déplore une indistinction moderne oblitérant la dualité ancienne. En inscrivant $a b$ ovo son projet dans la langue, Agamben court les risques philologiques déjà notés. Selon les protocoles d'administration de la preuve dans Homo sacer, les contre-exemples lexicaux affaiblissent une grande part de la démonstration. En effet, la prétendue nouveauté de l'époque moderne (cette "indifférence absolue », cette "zone d'indifférenciation irréductible » entre bios et zôê) n'est autorisée que par une version imaginaire de la langue. Laquelle langue, malgré le statut qu'elle paraît mériter dans l'éloge du logos politique, est ramenée, exemplairement, à une répartition de sens dénués de motilité, voire de vie (dans toutes les valeurs possibles du terme). Ici, le constat du « seul » mot de vie aujourd'hui mériterait un commentaire de plus. Qu'un unique substantif existe ne correspond pas à une indifférence; non plus que l'existence de parasynonymes ne se confond avec une répartition « claire ». Sommes-nous tous d'accord sur « ce que nous entendons par le mot vie » (p. 9)? Michel Henry, Gilles Deleuze, Jacques Derrida, français et contemporains, avaient-ils une seule ouïe? Le «terme unique » (p. 9) est aussi diffracté. Agamben n'explique pas la confusion actuelle par cette unicité lexicale. Et pour cause, la chronologie des événements biopolitiques serait bouleversée, depuis au moins le latin vita. Il y a lieu, pour Agamben, de préserver l'état d'exception d'une expérience grecque, où l'adéquation du langage à la situation politique serait par définition révélatrice. Cet extraordinaire statut du grec s'explique par son essence particulière. L'essence d'une langue chimériquement unifiée est posée en principe, échappant de ce fait à l'explication historique qu'elle suscite pourtant.

La distinction entre bios et zôê dans Homo sacer, et sa fortune nous apprennent donc beaucoup sur le projet d'Agamben. Elle situe la qualité de sa philologie, comme sa théorie lexicographique du sens. Le processus et les singularités de la signification sont fondus dans une rationalisation sémantique. Au nom du langage, on invente une langue dont la vie discursive est niée. Le succès de propagation d'une opposition bios/zôê fictive et figée nous en dit long sans doute aussi sur aujourd'hui. Il répond à une recherche contemporaine de légitimation philosophique grâce à 
l'apparat érudit des sciences humaines (la scientificité Foucault) - et aux limites de cette utilisation, finalement adventice (juxtaposée, ici, à un fonds originaire heideggerien). Mieux vaudrait, à l'inverse, une pratique jusqu'au-boutiste de l'enquête faisant sauter de l'intérieur les ligatures du concept. C'est dans ce parcours à nouveaux frais, par les textes du bios et de la $z \hat{o} \hat{e}$, que se trouverait quelque vie de la vie. 